\title{
Influência da prática habitual de ati- vidade física e da gordura de tronco sobre a glicemia de jejum em mulhe- res na menopausa
}

\section{Influence of habitual practice of physical activity and trunk fat on fasting glucose in postmenopausal women}

Camila Buonani ${ }^{1}$, Fabrício E Rossi ${ }^{1}$, Tiego A Diniz ${ }^{2}$, Diego G D Christofaro ${ }^{3}$, Rômulo A Fernandes ${ }^{3}$, Ismael F Freitas $\mathrm{Jr}^{4}$

\begin{abstract}
RESUMO
Modelo de estudo: Estudo Original

Objetivo: Examinar a relação entre a prática habitual de atividade física (AF) com a gordura corporal total, gordura do tronco e glicemia de jejum em mulheres na menopausa. Métodos: Foram avaliadas 60 mulheres na menopausa com média de idade de 61,0 anos, da cidade de Presidente Prudente - SP. A massa corporal (MC) e a estatura foram mensuradas para o cálculo do Índice de Massa Corporal (IMC). A gordura corporal total (GC) e a gordura de tronco (TrG) foram estimadas por meio do DEXA. A glicemia sanguínea foi dosada e classificada de acordo com os valores de referência da American Diabetes Association (1997). A prática habitual de AF foi estimada utilizando acelerômetro triaxial (Actigraph) e classificada de acordo com Sasaki (2010). Foi realizada a comparação entre mulheres com valores de glicemia normal e glicemia alterada por meio do teste de Kruskal-Wallis. Foi utilizado o coeficiente de correlação de Spearman e em seguida a regressão logística binária para verificar a associação entre nível de AF e composição corporal com glicemia de jejum. Resultados: Mulheres que apresentavam hiperglicemia obtiveram maiores valores de MC $(p=0,040)$, IMC $(p=0,002)$ e $G C(p=0,046)$, além de valores menores de AF leve $(p=0,019)$, moderada $(p=0,041)$ e de moderada+vigorosa $(p=0,041)$. A variável AF moderada e moderada+vigorosa apresentaram, respectivamente, correlação negativa significante com o percentual de GC ( $p=0,003$ e $p=0,003)$ e com $\operatorname{TrG}(p=0,021$ e $p=0,021)$, quando analisado os valores absolutos de GC foi observado correlação negativa com a soma moderada+vigorosa ( $p=0,049)$. As mulheres que obtiveram menores valores de $\operatorname{TrG}$ e que foram classificadas como suficientemente ativas apresentaram fator de proteção $(R C=0,133[I C=0,023-0,784])$ para glicemia de jejum alterada. Conclusão: A prática de AF de intensidade moderado-vigorosa, realizada pelo menos 150 minutos semanais, associado à baixa gordura de tronco, é um fator protetor para a hiperglicemia em mulheres na menopausa.
\end{abstract}

Palavras-chave: Atividade Motora. Hiperglicemia. Pós-menopausa. Acelerômetro.

1. Doutorandos em Ciências da Motricidade do Departamento de Educação Física - Universidade Estadual Paulista (UNESP), Campus de Rio Claro, São Paulo, Brasil.

2. Graduando do Curso de Educação Física - UNESP, Campus de Presidente Prudente, São Paulo, Brasil.

3. Professor Doutor do Departamento de Educação Física UNESP, Campus de Presidente Prudente, São Paulo, Brasil.

4. Livre Docente do Departamento de Educação Física - UNESP, Campus de Presidente Prudente, São Paulo, Brasil.
Correspondência: Camila Buonani Departamento de Educação Física Endereço: R. Roberto Simonsen, $305-$ CEP: $19060-900$ - Presidente Prudente - SP Telefone: (18) 3229.5828 Fax: (18) 3221.5681 camilabuonani@yahoo.com.br

Artigo recebido em 28/01/2013 Aprovado para publicação em 27/06/2013 


\section{Introdução}

O processo de envelhecimento é responsável por alterações significativas no organismo das quais colaboram para a diminuição da capacidade funcional e da aptidão física, que contribuem para a redução do nível de atividade física (AF). No caso da mulher, com a chegada da menopausa as consequências intrínsecas ao envelhecimento são acentuadas. ${ }^{1}$

Além disso, na menopausa é observado aumento da massa e da gordura corporal ${ }^{2}$, particularmente a central $^{3}$, fato que pode levar ao desenvolvimento de distúrbios metabólicos, como alterações da glicose sanguínea ${ }^{4}$, que desencadeia, via de regra, em diabetes mellitus tipo II (DM).

A DM é considerada uma das doenças mais prevalentes em mulheres na menopausa, e é um dos principais fatores de risco para a doença cardiovascular ${ }^{5}$, além de ser uma das maiores responsáveis pelo número de mortes nas populações ocidentais ${ }^{6}$.

Portanto, é importante a adoção de estratégias para reduzir fatores de risco para o desenvolvimento da DM e, nesse sentido, a prática suficiente de AF tem sido considerada uma estratégia interessante na prevenção dessa doença ${ }^{7}$, além da manutenção de baixa quantidade de gordura corporal ${ }^{4}$, no entanto, ainda não está claro qual desses componentes apresenta maior fator de proteção para evitar alterações da glicemia. Dessa forma, o objetivo do presente estudo foi examinar a relação entre a prática habitual de atividade física com a gordura do tronco e glicemia em mulheres na menopausa.

\section{Metodologia}

\section{Amostra}

Estudo de delineamento transversal foi realizado nos anos de 2011 e 2012, na cidade de Presidente Prudente - SP. A divulgação foi feita por meio da mídia local (jornais, televisão e rádio).

A amostra foi composta por 60 mulheres na menopausa com idade superior a 50 anos (média 61,0 $\pm 6,5$ anos) que concordaram participar de um programa de exercícios promovido pelo Departamento de Educação Física da Universidade Estadual Paulista (UNESP), Campus Presidente Prudente, SP, Brasil.

As mulheres foram consideradas aptas à participar do estudo se atendessem os seguintes critérios: i) estar na menopausa (não ter ciclo menstrual por um ano ou mais) ${ }^{8}$; ii) ter idade entre 50 e 79 anos na data da avaliação; iii) não ter praticado exercício físico nos últimos seis meses; iv) não fazer tratamento para reposição hormonal; v) assinar o termo de consentimento e esclarecimento formal para participação no estudo.

O presente estudo foi aprovado pelo Comitê de Ética e Pesquisa da Faculdade de Ciência e Tecnologia da Universidade Estadual Paulista, Campus Presidente Prudente, SP, Brasil (Protocolo 64/2011).

\section{Coleta de Dados}

\section{Antropometria e Composição Corporal}

A massa corporal foi aferida com a utilização de uma balança mecânica da marca Filizola, com precisão de $0,1 \mathrm{~kg}$ e capacidade máxima de $180 \mathrm{~kg}$; e a sua estatura foi aferida com a utilização de um estadiômetro fixo da marca Sanny, com precisão de $0,1 \mathrm{~cm}$ e extensão máxima de dois metros, de acordo com a metodologia proposta por Freitas Jr. et al. ${ }^{9}$.

A composição corporal foi estimada por meio da técnica de Dual-energy X-ray absorptiometry (DEXA [Lunar brand, DPX-MD, software 4.7]), da qual foi utilizada para mensurar a gordura corporal total, expressas em kg e percentuais, e a gordura de tronco, expressa em kg.

\section{Análise da glicemia sérica}

A análise da glicemia foi realizada em laboratório especializado, no qual foi respeitado um jejum de 12 horas. A coleta foi realizada em tubo à vácuo com gel separador sem anticoagulante; após a coleta, o sangue foi centrifugado por 10 minutos a $3.000 \mathrm{rpm}$ para separar o soro dos demais componentes do sangue, sendo que o soro foi utilizado para as análises.

Para dosagem da glicemia utilizou-se um kit enzimático colorimétrico processado em um aparelho Autohumalyzer. ${ }^{10}$ As anormalidades dos valores de glicemia foram definidas de acordo com o valor de referência propostos pela American Diabetes Association. ${ }^{11}$

\section{Acelerômetro GT3X da Actigraph}

Para análise da prática habitual de AF, foi utilizado um sensor de movimento tipo acelerômetro triaxial marca Actigraph, modelo GT3X (Actigraph LLC, Pensacola, FL). Os aparelhos são leves (27 gramas) e pequenos (dimensões de $3,8 \mathrm{~cm}$ x $3,7 \mathrm{~cm}$ x 1,8 $\mathrm{cm}$ ), e são desenhados para registrar os movimentos nos três planos ortogonais: vertical, horizontal anteroposterior e médio-lateral. O ActiGraph GT3X 
mensura e registra variações de aceleração cujas magnitudes abrangem aproximadamente 0,05 e 2,5 G $\left(1 \mathrm{G}=9,8 \mathrm{~m} / \mathrm{s}^{2}\right)$ dentro de uma faixa de frequência de 0,25 a 2,5 Hertz. A saída do acelerômetro é digitalizada por um conversor Analógico-Digital de 12 bits a uma taxa de 30 vezes por segundo (30 Hertz).

Cada amostra dos registros (counts) foi resumida ao longo de um intervalo específico de tempo (epoch), de 60 segundos. Para população desse estudo optou-se por um minuto, pois o tipo de AF dos mesmos relaciona-se com o padrão de atividade de baixa intensidade e longa duração. ${ }^{12}$

Os acelerômetros foram colocados, presos na cintura dos avaliados, que permaneceram com o equipamento por oito dias, dos quais, sete foram dias completos. Instruções para utilização do aparelho foram realizadas previamente. $\mathrm{O}$ acelerômetro deveria ser utilizado o dia inteiro durante as horas em que o indivíduo permanece em vigília, sendo retirado apenas quando houvesse contato com água (higiene pessoal ou atividades aquáticas).

Para análise dos dados foi utilizado o software específico, ActiLife5 - Data Analysis Software by Actigraph. Foram incluídos no banco de dados apenas dias completos de monitoramento. Hora consecutiva de zero counts foram considerados como período em que o paciente não estava utilizando o acelerômetro e dias com menos de dez horas em utilização do aparelho foram excluídos, pelo aumento da variabilidade. ${ }^{13}$ Ao final, cada sujeito do estudo deveria ter, no mínimo, cinco dias completo de monitoramento, número de dias requeridos para estimar as variáveis normalmente utilizadas em estudos com acelerômetro. ${ }^{12}$

A intensidade da prática de AF foi analisada de acordo com a recomendação estabelecida por Sasaki et al. ${ }^{14}$ para acelerômetros triaxiais, da qual determina que AF leve ( $<3,00 \mathrm{METs})$ é definida como menos de 2690 counts por minuto, AF moderada é definida como os counts entre 2690 e 6166 (3,00-5,99 METs), a AF vigorosa é definida como os valores de counts entre o intervalo de 6167 a 9642 (6,00-8,99 METs), e a AF muito vigorosa é compreendida em valores superiores a 9642 counts por minuto ( $\geq$ METs).

A amostra foi estratificada de acordo com os minutos de AF moderado-vigorosa; mulheres que não cumpriram ao menos 150 minutos de AF moderadovigorosa por semana foram consideradas insuficientemente ativas, e mulheres que cumpriram essas recomendações, suficientemente ativas. ${ }^{15}$ Os valores foram expressos em minutos/semana de AF leve, moderada e vigorosa e counts totais de AF (valores brutos, estabelecidos por meio da magnitude dos 3 eixos de vetores).

\section{Caracterização dos Fatores de Risco para glicemia alterada}

A identificação dos fatores de risco foi feita a partir das medidas de gordura de tronco e da prática habitual de AF. A gordura de tronco não possui valor de referência, dessa forma, a partir dos valores absolutos obtidos, a amostra foi dividida em tercis, e foi considerado excesso de gordura no tronco, os valores posicionados no tercil superior, e como pouca gordura de tronco, os valores que se encontravam no $1^{\circ}$ e $2^{\circ}$ tercil.

O nível de AF foi classificado de acordo com as recomendações publicadas por Sasaki et al. ${ }^{14} \mathrm{e}$ as participantes foram estratificadas em dois grupos, o que atendiam as recomendações de AF do American College of Sports Medicine and the American Heart Association ${ }^{15}$ (mais que 150 minutos semanais de AF moderado-vigorosa), denominadas suficientemente ativas, e as que não atendiam, consideradas insuficientemente ativas.

\section{Análise Estatística}

A normalidade foi verificada por meio do teste de Kolmogorov-Smirnov $(K-S)$ para averiguar o enquadramento dos dados no modelo Gaussiano de distribuição. A apresentação dos resultados foi feita pela estatística descritiva, expressos em mediana, intervalo interquartil e intervalo de confiança de $95 \%$. A comparação das variáveis contínuas entre mulheres com valores de glicemia normal e glicemia alterada foi realizada por meio do teste de Kruskal-Wallis. Foi utilizada o coeficiente de correlação de Spearman para verificar a associação entre nível de AF e composição corporal com glicemia de jejum. Para verificar a associação entre a prática habitual de atividade física, percentual de gordura e os valores de glicemia em mulheres menopausadas foi utilizada a regressão logística binária.

Todas as análises foram realizadas utilizando o programa SPSS, versão 17.0 (SPSS Inc, Chicago, IL) e a significância estatística foi estabelecida em 5\%.

\section{Resultados}

As características gerais da amostra com dados de composição corporal, antropometria e nível de 
atividade física estão apresentadas na Tabela 1. Na amostra estudada foi observado que $28,3 \%$ das participantes foram consideradas hiperglicêmicas, 31,7\% foram classificadas como insuficientemente ativas.
Na Tabela 2 são apresentados os resultados das comparações realizadas entre as mulheres que obtiveram glicemia normal e alterada. Foi verificado que $28,3 \%$ apresentaram valores de glicemia de jejum

Tabela 1

Características gerais da amostra $(n=60)$

\begin{tabular}{|c|c|c|c|c|c|}
\hline \multirow[b]{2}{*}{ Idade (anos) } & \multicolumn{2}{|c|}{ Mediana (IR) } & \multicolumn{3}{|c|}{ (IC 95\%) } \\
\hline & 60,8 & $(8,9)$ & 59,5 & - & 62,9 \\
\hline Peso (kg) & 66,7 & $(16,1)$ & 66,1 & - & 73,4 \\
\hline Estatura $(\mathrm{cm})$ & 154,4 & $(9,0)$ & 154,5 & - & 158,3 \\
\hline $\operatorname{IMC}\left(\mathrm{kg} / \mathrm{m}^{2}\right)$ & 28,1 & $(7,1)$ & 27,1 & - & 30,9 \\
\hline $\mathrm{GC}(\mathrm{Kg})$ & 28,8 & $(11,9)$ & 28,5 & - & 33,5 \\
\hline $\mathrm{GC}(\%)$ & 44,2 & $(10,0)$ & 42,2 & - & 45,4 \\
\hline Gordura de Tronco (Kg) & 22,2 & $(4,8)$ & 21,9 & - & 23,9 \\
\hline Glicemia (mg/dL) & 90,0 & $(19,0)$ & 92,4 & - & 108,1 \\
\hline \multicolumn{6}{|l|}{ Variáveis do Acelerômetro } \\
\hline AF Sedentária (min/sem) & 6323,6 & $(1349,0)$ & 6228,7 & - & 6718,7 \\
\hline AF leve (min/sem) & 2193,4 & $(736,1)$ & 1929,1 & & 2188,1 \\
\hline AF moderada (min/sem) & 136,1 & $(133,8)$ & 125,4 & - & 184,0 \\
\hline AF Vigorosa (min/sem) & 0,0 & $(0,0)$ & 0,0 & - & 4,4 \\
\hline Soma AF Moderado-Vigorosa (min/sem) & 136,1 & $(133,8)$ & 125,7 & - & 187,4 \\
\hline
\end{tabular}

$\mathrm{IR}=$ intervalo Interquartil; IC95\%= intervalo de confiança de 95\%; IMC= índice de massa corporal; GC= gordura corporal; $\mathrm{AF}=$ atividade física; $\mathrm{min} / \mathrm{sem}=$ minutos por semana .

Tabela 2

Valores de mediana e intervalo interquartil de atividade física e composição corporal de acordo com a glicemia

\begin{tabular}{|c|c|c|c|}
\hline & $\begin{array}{c}\text { Glicemia Normal } \\
\qquad(\mathrm{N}=\mathbf{4 3})\end{array}$ & $\begin{array}{c}\text { Glicemia Alterada } \\
\qquad(\mathbf{N}=\mathbf{1 7})\end{array}$ & $\mathbf{P}$ \\
\hline Idade (anos) & $60,6 \quad(7,5)$ & $64,9 \quad(11,3)$ & 0,133 \\
\hline Peso (kg) & $65,6 \quad(12,4)$ & $72,3 \quad(19,2)$ & 0,040 \\
\hline Estatura $(\mathrm{cm})$ & $158,4 \quad(8,0)$ & 151,5 & 0,001 \\
\hline $\mathrm{IMC}\left(\mathrm{kg} / \mathrm{m}^{2}\right)$ & $26,1 \quad(5,4)$ & $(9,7)$ & 0,002 \\
\hline GC (kg) & $27,4 \quad(11,0)$ & $33,2 \quad(16,8)$ & 0,046 \\
\hline $\mathrm{GC}(\%)$ & $42,0 \quad(10,1)$ & $(7,3)$ & 0,121 \\
\hline Gordura de Tronco (kg) & $22,1 \quad(4,5)$ & $(5,8)$ & 0,096 \\
\hline \multicolumn{4}{|l|}{ Variáveis do Acelerômetro } \\
\hline AF Sedentária (min/sem) & $6308,9(1267,9)$ & $6696,0(1831,1)$ & 0,646 \\
\hline AF Leve (min/sem) & $2232,1 \quad(658,5)$ & $1741,8 \quad(841,5)$ & 0,019 \\
\hline AF Moderada (min/sem) & $147,2 \quad(142,8)$ & $101,1 \quad(140,6)$ & 0,041 \\
\hline AF Vigorosa (min/sem) & $(1,0)$ & $(0,0)$ & 0,061 \\
\hline Moderada + Vigorosa (min/sem) & $147,2 \quad(142,6)$ & $101,1 \quad(140,6)$ & 0,041 \\
\hline
\end{tabular}

Nota: $\mathrm{AF}=$ atividade física; $\mathrm{kg}=$ quilogramas; $\%=$ percentual $; \mathrm{min} / \mathrm{sem}=$ minutos por semana. 
acima do recomendado. Ademais, observou-se que as mulheres que apresentavam hiperglicemia obtiveram estatisticamente maiores valores de massa corporal $(\mathrm{p}=0,040), \operatorname{IMC}(\mathrm{p}=0,002)$ e gordura corporal total $(\mathrm{p}=0,046)$, além de valores menores de AF leve $(\mathrm{p}=0,019)$, moderada $(\mathrm{p}=0,041)$ e do somatório de moderada e vigorosa $(\mathrm{p}=0,041)$.

Na Tabela 3 estão apresentados os valores de correlação entre composição corporal e nível de AF. A análise do nível de AF moderada e a soma moderada+vigorosa apresentaram, respectivamente, correlação negativa significante com o percentual de gordura total $(\mathrm{p}=0,003$ e $\mathrm{p}=0,003)$ e com gordura de tronco $(\mathrm{p}=0,021$ e $\mathrm{p}=0,021)$, quando analisado os valores absolutos de gordura corporal total foi observado correlação negativa com a soma moderada+vigorosa $(\mathrm{p}=0,049)$.

Na Figura 1 são apresentados os resultados da regressão múltipla modelada pelo nível de AF, gordura de tronco e hiperglicemia. As mulheres que ou eram suficientemente ativas ou tinham baixa gordura de tronco não apresentaram correlação estatística aos

Tabela 3

Correlações entre as variáveis de atividade física com composição corporal e glicemia de jejum

\begin{tabular}{lcccc}
\hline & $\mathrm{GC}$ & $\% \mathrm{GC}$ & $\mathrm{G}-$ Tronco & \multicolumn{2}{c}{ Glicemia } \\
\cline { 2 - 5 } Atividade física habitual & $\mathrm{R}$ & $\mathrm{R}$ & $\mathrm{R}$ & $\mathrm{R}$ \\
\hline PIAF $(\mathrm{min} / \mathrm{sem})$ & $-0,460$ & $-0,199$ & $-0,143$ & $-0,010$ \\
Leve $(\mathrm{min} / \mathrm{sem})$ & $-0,254$ & $-0,213$ & $-0,251$ & $-0,250$ \\
Moderada $(\mathrm{min} / \mathrm{sem})$ & $-0,252$ & $-0,382^{* *}$ & $-0,298^{*}$ & $-0,166$ \\
Vigorosa $(\mathrm{min} / \mathrm{sem})$ & $-0,194$ & $-0,231$ & $-0,187$ & $-0,101$ \\
Moderada + Vigorosa (min/sem) & $-0,255^{*}$ & $-0,381^{*}$ & $-0,298^{*}$ & $-0,166$ \\
\hline
\end{tabular}

$\mathrm{GC}=$ Gordura corporal; G_Tronco = gordura de tronco; $\mathrm{R}=$ coeficiente de correlação; PIAF= Prática Insuficiente de Atividade Física; $\mathrm{min} / \mathrm{sem}=$ minutos por semana. ${ }^{*} \mathrm{p}<0,05 ; * * \mathrm{p}<0,01$

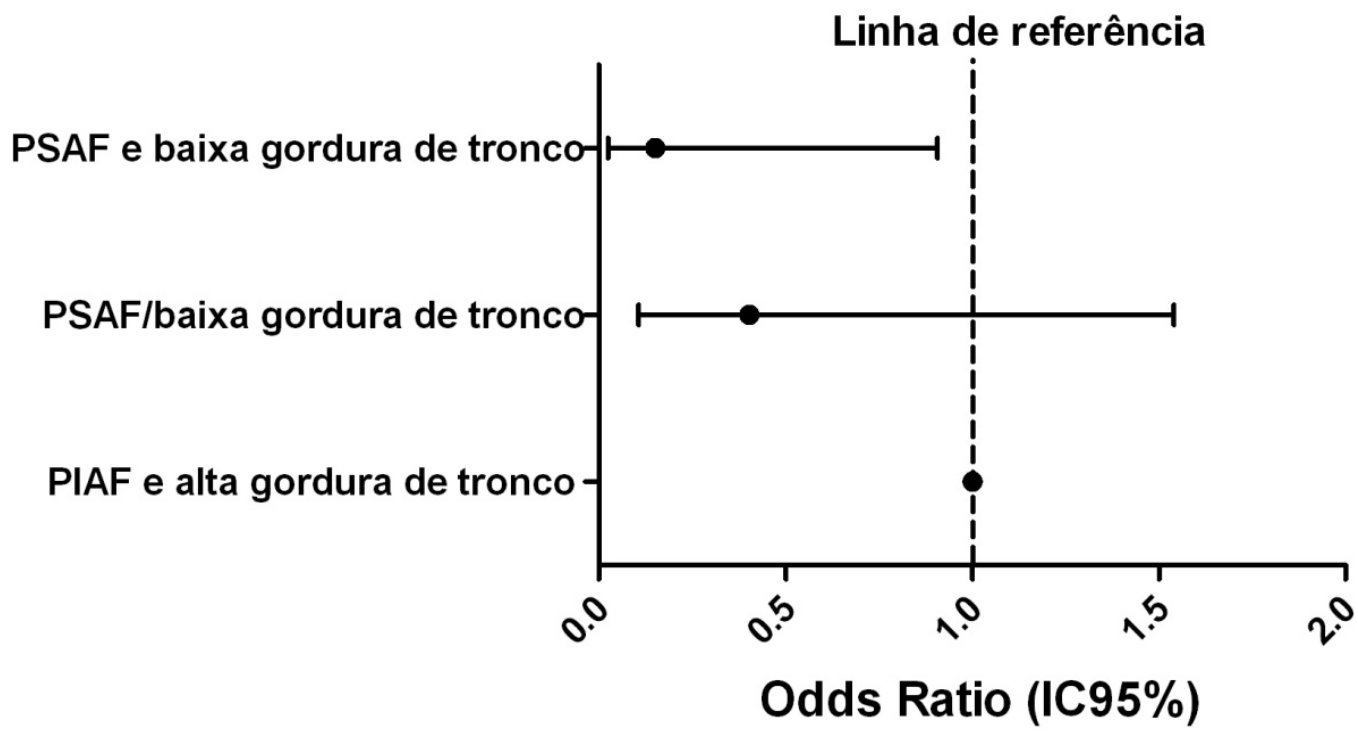

Figura 1. Associação entre Prática habitual de atividade física, gordura de tronco e glicemia alterada de mulheres com menopausa, Presidente-Prudente-SP, 2012.

Nota: PSAF = Prática suficiente de atividade física; PIAF = Prática insuficiente de atividade física; PSAF/baixa gordura de tron $\mathrm{C}=$ mulheres que tinham um dos dois fatores apenas, ou a prática suficiente de atividade física ou a baixa gordura de tronco. 
valores de glicemia (respectivamente, Razão de Chance $=0,350$ [IC=0,095-1,290]). Porém foi observado que as mulheres que obtiveram menores valores de gordura de tronco e que foram classificadas como suficientemente ativas apresentaram fator de proteção (Razão de Chance=0,133 [IC=0,023-0,784]) para glicemia de jejum alterada.

\section{Discussão}

Estudo de delineamento transversal teve como objetivo observar as possíveis associações entre a prática habitual de AF e composição corporal, com valores alterados de glicemia sanguínea de mulheres na menopausa. Verificou-se que a AF moderada e a AF moderado-vigorosa apresentaram relação negativa significativa com percentual de gordura corporal e gordura de tronco. Foi observado que as participantes que apresentaram glicemia de jejum aumentada obtiveram maiores valores de massa corporal, de IMC e gordura corporal (total e de tronco), e alcançaram menores valores de AF leve, moderada e a moderado- vigorosa. As mulheres que, simultaneamente, obtiveram menores valores de gordura de tronco e foram classificadas como suficientemente ativas apresentaram fator de proteção para glicemia alterada.

A menopausa é caracterizada como um período no qual ocorrem importantes alterações na composição corporal, como o aumento da gordura corporal, principalmente na região do tronco ${ }^{3}$, podendo acarretar em aumento de disfunções metabólicas, como hiperglicemia e DM. ${ }^{2}$

Além desses fatores de risco, sabe-se que o nível de AF das pessoas diminui com o envelhecimento, e isso ocorre independente do sexo. Portanto, o sedentarismo caracterizado por atividades com baixo gasto energético, como elevado número de horas assistindo televisão e/ou usando o computador, das quais fazem parte dos hábitos cotidianos de grande parte da população pode agravar as consequências intrínsecas ao envelhecimento. ${ }^{16}$

No presente estudo, verificou-se que a AF moderada e a AF moderado+vigorosa, apresentaram correlação negativa com percentual de gordura corporal e gordura de tronco. Foi observado também que as participantes que apresentaram glicemia de jejum aumentada, obtiveram maiores valores de massa corporal, IMC e gordura corporal total e de tronco, e, também, menores valores de AF leve, moderada e moderado+vigorosa.
As mulheres classificadas como suficientemente ativas e com menor quantidade de gordura de tronco apresentaram menor chance de desenvolvimento de DM.

Em estudo realizado por Sternfeld et al. ${ }^{17}$, com 459 mulheres norte-americanas também verificou uma relação inversa, entre AF total e percentual de gordura corporal, quando ajustado pela idade, reforçando os achados do presente estudo. A investigação de Colpani et al. ${ }^{7}$, constatou que mulheres na menopausa que foram consideradas insuficientemente ativas, avaliadas por meio de pedômetro, apresentaram risco aumentado para o desenvolvimento de DM e síndrome metabólica. Resultados esses que corroboram com a presente investigação.

Ratificando a importância da prática de AF, Kirk et al. ${ }^{18}$ verificaram que indivíduos com DM e que aumentaram o nível de AF, avaliados por acelerometria biaxial, apresentaram melhora do controle glicêmico e redução de risco cardiovascular. Adicionalmente, Healy et al. ${ }^{19}$, investigaram o nível de AF, também por meio da acelerometria biaxial, de indivíduos sem diagnóstico de DM e constatou que menor tolerância á glicose apresentou correlação positiva com AF insuficiente e correlação negativa com tempo de prática de AF leve e a soma de moderada+vigorosa.

O estado hiperglicêmico é um forte indicativo de menor sensibilidade à ação da insulina. Há evidências que indivíduos com níveis glicêmicos alterados podem desenvolver DM em um período de cinco a 10 $\operatorname{anos}^{20}$, além disso, a hiperglicemia está associada com aumento de risco de doença cardiovascular e morte prematura. $^{5,21}$

Após a comparação da amostra do presente estudo de acordo com o estado hiperglicêmico (Tabela 2), foi observado que o grupo de mulheres que apresentou glicemia de jejum alterada obteve valores inferiores em todas as variáveis de AF. Em contrapartida, quando analisada a gordura corporal, esse grupo apresentou valores estatisticamente maiores.

Os resultados encontrados sugerem que mulheres na menopausa que são suficientemente ativas e têm valores menores de gordura no tronco apresentam fator de proteção para o desenvolvimento de hiperglicemia (Figura 1). A AF pode exercer grande influência para a manutenção da glicemia em valores considerados normais, visto que a prática de AF constante contribui para a estimulação da captação da glicose pelo GLUT4 ${ }^{22}$, reduzindo o nível de glicose sanguínea e, assim, contribuindo para a estabilização da mesma. 
Sabe-se que o ganho de peso, a baixa capacidade cardiorrespiratória, a força muscular diminuída e o sedentarismo são alguns dos principais fatores associados à maior prevalência de doenças crônicodegenerativas, entre elas está a DM, em mulheres na menopausa $^{23}$, indicando a necessidade de se adotar estratégias para minimizar ou reverter esse quadro.

A importância da prática de AF para indivíduos com DM não se restringe ao aspecto metabólico e de saúde física, mas envolve a condição econômica, pois em estudo recente realizado por Codogno et al. ${ }^{24}$, foi verificado que indivíduos com DM com menor prática de AF apresentaram gastos estatisticamente maiores com consultas e com compra de medicamentos, quando comparados àqueles que praticavam mais AF.

Apesar da importância dos achados do presente estudo é necessário mencionar suas limitações. Primeiro, o tamanho amostral reduzido que indica a necessidade de cautela na extrapolação dos resultados. Segundo, o desenho transversal do estudo que dificulta a análise sobre o efeito da prática de AF, nesse sentindo sugere-se estudos longitudinais de intervenção para verificar o efeito da prática habitual de AF na composição corporal em mulheres na menopausa.

Entretanto, devem-se ressaltar alguns aspectos positivos do presente estudo. Talvez o mais proe- minente seja a mensuração da AF de maneira objetiva por meio do acelerômetro triaxial, evitando, assim, o erro da medição de auto-relato, que normalmente é utilizada, uma vez que em idosos, as memórias de curto e longo prazo são limitadas, e dificilmente apresentam percepção do que significa uma AF leve, moderada ou vigorosa. ${ }^{25}$ Adicionalmente, a utilização do DEXA para estimar a composição corporal é outro aspecto diferencial, visto que é um método de alta precisão e fidedigno para a população idosa e que também fornece resultados de composição corporal dos segmentos do corpo. ${ }^{26}$

Em síntese, os resultados aqui observados sugerem que a prática de AF de intensidade moderadovigorosa, realizada pelo menos 150 minutos semanais, associado à baixa gordura de tronco, é um fator protetor para a hiperglicemia em mulheres na menopausa, resultando assim em menor risco de desenvolver doenças cardiovasculares e de morte prematura. Dessa forma, estratégias que tenham como objetivo a manutenção de valores glicêmicos considerados ideais em mulheres na menopausa, devem focar no aumento da prática de AF e controle da intensidade dessa variável, conjuntamente com a redução da gordura corporal na região central.

Agradecimentos: Capes, UnATI e Athia.

\begin{abstract}
:
Study Model: Original Study

Objective: To examine the relationship between the practice of physical activity (PA) with total body fat, trunk fat and fasting glucose in postmenopausal women. Methods: Sixty postmenopausal women, mean age of 61.0 years, from the city of Presidente Prudente SP, were assessed. Body mass (BM) and height were measured to calculate Body Mass Index (BMI). Total body fat (BF) and trunk fat mass (TFM) were estimated using DEXA. Fasting glucose was measured and classified according to the reference values of the American Diabetes Association (1997). The usual practice of PA was estimated using triaxial accelerometer, model GT3X (Actigraph,Pensacola,FL,USA) and classified according to Sasaki (2010). Comparisons were made between women with normal blood fasting glucose levels (NBG) and high blood fasting glucose $(\mathrm{HBG})$ levels by the Kruskal-Wallis test. Spearman correlation coefficient was used and then the binary logistic regression to test the association between PA level and body composition with fasting glucose. Results: Women with HBG showed higher values of BM $(p=0.040)$, BMI $(p=0.002)$ and $B F(p=0.046)$, and lower values of light $(p=0.019)$, moderate $(p=0.041)$ and moderate + vigorous $(p=0.041)$ PA. The moderate and moderate+vigorous PA showed, respectively, a significant negative correlation with BF ( $p=0.003$ and $p=0.003)$ and TFM $(p=0.021$ and $p=0.021)$. The FM presented negative correlation with moderate+vigorous $(p=0.049)$. Women with lower TFM and classified as active showed protective factor $(\mathrm{OR}=0.133$ [ $\mathrm{Cl} 0.023$ to 0.784$]$ ) for impaired fasting glucose. Conclusion: The practice of moderate+vigorous intensity PA, held at least 150 minutes per week, is associated with lower trunk fat, and presents protective factor for impaired fasting glucose and risk of diabetes mellitus in postmenopausal women.
\end{abstract}

Keywords: Physical Activity. Hyperglycemia. Postmenopause. Accelerometer. 


\section{Referências}

1- Whitcroft S, Herriot A. Insulin resistance and management of the menopause: a clinical hypothesis in practice. Menopause Int. 2011;17:24-8.

2- Mazo GZ, Liposcki DB, Ananda C, Preve D. Condições de saúde, incidência de quedas e nível de atividade física dos idosos. Rev Bras Fisioter. 2007;11:437-42.

3- Donato GB, Fuchs SC, Oppermann K, Bastos C, Spritzer PM. Association between menopause status and central adiposity measured at different cutoffs of waist circumference and waist-to-hip ratio. Menopause. 2006;13:280-5.

4- Mokdad AH, Stroup DF, Giles WH. Public health surveillance for behavioral risk factors in a changing environment. Recommendations from the Behavioral Risk Factor Surveillance Team. MMWR Recomm Rep.2003;52:1-12.

5- Schramm TK, Gislason GH, Kober L, Rasmussen S, Rasmussen JN, Abildstrom SZ, et al. Diabetes patients requiring glucose-lowering therapy and nondiabetics with a prior myocardial infarction carry the same cardiovascular risk: a population study of 3.3 million people. Circulation. 2008;117:1945-54.

6- Office for National Statistics Mortality statistics cause review of the registrar general on deaths by cause, sex and age, in England and Wales, London 2003; Series DH2 (30).

7- Colpani V, Oppermann K, Bastos C, Spritzer PM. Association between habitual physical activity and lower cardiovascular risk in premenopausal, perimenopausal, and postmenopausal women: a population-based study. Menopause. 2013; 20(5). Em publicação 2013.

8- ORGANIZACIÓN MUNDIAL DE LA SALUD. Investigaciones sobre la menopausia en los años noventa. Genebra: Organización Mundial de la Salud; 1996

9- Freitas Jr IF et. al. Padronização de Técnicas Antropométricas. Ed Cultura Acadêmica (Presidente Prudente, SP); 2009.

10- Human RP, Jones GA. Evaluation of swab transport systems against a published standard. J Clin Pathol. 2004;57:762-3.

11- Expert Committee on the Diagnosis and Classification of Diabetes Mellitus: Report of the Expert Committee on the Diagnosis and Classification of Diabetes Mellitus. Diabetes Care. 1997;20:1183-97.

12- Trost SG, Mclver KL, Pate RR. Conducting accelerometerbased activity assessments in field-based research. Med Sci Sports Exerc. 2005;37(11 Suppl):S531-43.

13- Craig CL, Marshall AL, Sjostrom M, Bauman AE, Booth ML, Ainsworth BE, et al. International physical activity questionnaire: 12 -country reliability and validity. Med Sci Sports Exerc. 2003;35:1381-95.
14- Sasaki JE, John D, Freedson PS. Calibration of the Actigraph GT3X Activity Monitor. International Congress on Physical Activity and Public Health (ICPAPH). 2010.

15- Haskell WL, Lee IM, Pate RR, Powell KE, Blair SN, Franklin BA, et al.: Physical activity and public health: updated recommendation for adults from the American College of Sports Medicine and the American Heart Association. Med Sci Sports Exerc. 2007;39:1423-34.

16- Ford K, Sowers M, Seeman TE, Greendale GA, Sternfeld B, Everson-Rose SA. Cognitive functioning is related to physical functioning in a longitudinal study of women at midlife. Gerontology. 2010;56:250-8.

17- Sternfeld B, Bhat AK, Wang H, Sharp T, Quesenberry CP, Jr. Menopause, physical activity, and body composition/fat distribution in midlife women. Med Sci Sports Exerc. 2005; 37: 1195-202.

18- Kirk A, Mutrie N, Maclntyre P, Fisher M. Increasing physical activity in people with type 2 diabetes. Diabetes Care. 2003;26:1186-92

19- Healy GN, Dunstan DW, Salmon J, Cerin E, Shaw JE, Zimmet $P Z$, et al. Objectively measured light-intensity physical activity is independently associated with 2-h plasma glucose. Diabetes Care. 2007;30:1384-9.

20- Torres-Leal FL, Capitani MD, Tirapegui J. The effect of physical exercise and caloric restriction on the components of metabolic syndrome. Braz J Pharm Sci. 2009;45:379-99.

21- Haffner SM, Lehto S, Ronnemaa T, Pyorala K, Laakso M. Mortality from coronary heart disease in subjects with type 2 diabetes and in nondiabetic subjects with and without prior myocardial infarction. N Engl J Med. 1998;339:229-34.

22- Hayashi T, Wojtaszewski JF, Goodyear LJ. Exercise regulation of glucose transport in skeletal muscle. Am J Physiol. 1997;273(6 Pt 1):E1039-51.

23- Knopp RH. Risk factors for coronary artery disease in women. Am J Cardiol. 2002;89(12A):28E-34E; discussion 34E-35E.

24- Codogno JS, Fernandes RA, Monteiro, HL. Prática de atividades físicas e custo do tratamento ambulatorial de diabéticos tipo 2 atendidos em unidade básica de saúde. Arq Bras Endocrinol Metab. 2012;56/1

25- Shephard RJ. Limits to the measurement of habitual physical activity by questionnaires. Br J Sports Med 2003;37:197206; discussion 206.

26- Woodrow G. Body composition analysis techniques in the aged adult: indications and limitations. Curr Opin Clin Nutr Metab Care 2009;12:8-14 